\title{
Oral paricalcitol for the treatment of secondary hyperparathyroidism in chronic kidney disease
}

\author{
Steven Cheng \\ Daniel Coyne \\ Division of Nephrology, Washington \\ University, School of Medicine, Saint \\ Louis, MO, USA
}

\begin{abstract}
Secondary hyperparathyroidism (SHPT) is commonly seen in patients with chronic kidney disease (CKD). Even in early CKD, parathyroid hormone (PTH) levels are elevated, maintaining mineral homeostasis at the cost of long-term bone health. One potent stimulus for PTH secretion is a deficiency of active vitamin D. Replacement with calcitriol, the active form of vitamin D, lowers PTH but often raises calcium and phosphorus levels, predisposing patients to an increased risk of ectopic calcifications. Paricalcitol is a vitamin D analog designed to treat SHPT without raising serum calcium and phosphorus levels. The intravenous preparation of paricalcitol is used routinely in the hemodialysis population and has demonstrated a survival benefit over calcitriol in hemodialysis patients. A new oral preparation has now been developed for use in the pre-dialysis CKD population. Thus far, oral paricalcitol has been shown to reduce PTH by an average of $42 \%$ in CKD patients, while having minimal effects on serum calcium and phosphorus. While long term effects of the oral preparation have yet to be studied, emerging evidence suggests that paricalcitol mediates a variety of beneficial effects through the activation of vitamin D receptors which may result in improved survival.
\end{abstract}

Keywords: paricalcitol, secondary hyperparathyroidism, chronic kidney disease

\section{Introduction}

Secondary hyperparathyroidism (SHPT) is a serious complication of chronic kidney disease (CKD). While overt manifestations such as pain, weakness, pruritis, and metastatic calcifications may not occur until late in CKD or at end stage renal disease, the disease process begins early in the course of CKD, at glomerular filtration rates of about $60 \mathrm{ml} / \mathrm{min} / 1.73 \mathrm{~m}^{2}$ (Pitts et al 1988; Reichel et al 1991). Approximately 7 million Americans have kidney function at or below this level. Declining kidney function leads to a deficiency in active vitamin D, or calcitriol, which plays a critical role in the pathogenesis of SHPT and its associated cardiovascular complications. However, treatment with calcitriol is limited by its hypercalcemic and hyperphosphatemic effects. These side effects promote ectopic, and especially vascular, calcifications which increase cardiovascular risk (Qunibi et al 2002; Goodman et al 2003; Goldsmith et al 2004). Vitamin D analogs, like paricalcitol, have been developed to suppress parathyroid hormone (PTH) secretion without concomitant elevations in the serum calcium or phosphorus levels. This article will review the pathophysiology of SHPT, the data on paricalcitol for the treatment of SHPT, and the potential long term benefits of early oral administration of this medication.

\section{Calcitriol deficiency in SHPT}

Calcitriol deficiency plays a central role in the pathogenesis of SHPT. The decline in functional renal mass leads to a decrease in the 1-alpha hydroxylation of vitamin D 
to its active form, calcitriol. Under normal conditions, calcitriol regulates the expression of PTH by suppressing transcription of pre-pro-PTH messenger RNA (Russell et al 1986; Silver et al 1986). Thus decreases in calcitriol levels result in decreased activation of vitamin $D$ receptors, leading to increased expression of PTH and hyperplastic growth of the parathyroid gland. Furthermore, calcitriol deficiency results in decreased intestinal absorption of calcium. In response to the fall in serum calcium levels, PTH secretion increases as a compensatory mechanism to maintain mineral homeostasis.

Calcitriol deficiency also decreases the activation of other vitamin D receptors distributed throughout the body, including the intestines, kidneys, bone, immune cells, skin, heart, and brain (Mee et al 1996; Dusso and Brown 1998), leading to a variety of adverse effects associated with SHPT. At normal levels, calcitriol has a protective effect against vascular calcifications. Activation of the vitamin D receptor (VDR) on vascular smooth muscle cells has been found to regulate the synthesis of type 1 collagen, core binding factor alpha and bone morphogenic protein 2 - all of which contribute to the development of arterial calcification and mineralization (Virdi et al 1998; Bellows et al 1999; Drissi et al 2002). Calcitriol may play an important role in preventing atherosclerosis as well. Activation of the VDR stimulates the protective effects of type 2 helper T (Th2) cells through the induction of cytokines such as IL4 and IL10 (Boonstra et al 2001; Barrat et al 2002). In addition, calcitriol decreases interferon (IFN) gamma, which is an important component of macrophage recruitment in the pathogenesis of atherosclerotic lesions (Staeva-Vieira and Freedman 2002). Calcitriol deficiency in renal failure decreases VDR activation and may thus play a direct role in mediating cardiovascular complications.

\section{Treatment}

The treatment of SHPT early in CKD involves some form of active vitamin $\mathrm{D}$ along with dietary phosphorus restriction and phosphorus-binding agents. Active vitamin D supplementation with calcitriol has been shown to reduce serum PTH levels and bone turnover in both animal studies and in clinical trials (Slatopolsky et al 1984). However, the administration of calcitriol also stimulates VDRs that increase intestinal absorption of calcium and phosphorus and suppress normal bone turnover, leading to elevations in the calcium and phosphorus levels. Hypercalcemia and hyperphosphatemia are associated with ectopic calcifications and an increase in cardiovascular-related deaths in dialysis patients (Qunibi et al 2002; Goodman et al 2003; Goldsmith et al 2004). The process of vascular calcification appears to begin in CKD as evidenced by the data from the Dallas Heart Study and a recent study by Sigris and colleagues (Kramer et al 2005; Sigris et al 2006). Furthermore, the increasing calcium burden places the patient in chronic positive calcium balance. Therefore, while the administration of calcitriol is certainly effective, elevations in serum calcium and phosphorus need to be monitored regularly and the dose of calcitriol may be limited by increases in these divalent ions.

Analogs of active vitamin D were developed to inhibit parathyroid secretion while minimizing the effects of vitamin D receptor activation elsewhere. Paricalcitol, 19nor- $1,25(\mathrm{OH})_{2} \mathrm{D}_{2}$, is the most commonly used of these active vitamin $\mathrm{D}$ analogs. It achieves its site selectivity via removal of a methylene group from the carbon at the 19 position, and a $\mathrm{D}_{2}$ side chain. While intravenous paricalcitol has been used in dialysis patients since its approval in 1998, the oral formulation has only recently been approved. Thus the majority of studies evaluating the effectiveness of paricalcitol, especially in comparison with other formulations of active vitamin $\mathrm{D}$, have been done with the intravenous form in patients with end-stage renal disease on dialysis. Like calcitriol, paricalcitol suppresses pre-proPTH mRNA synthesis and has a prolonged effect of PTH inhibition beyond its mean half-life of approximately 14 hours in hemodialysis patients (Cato et al 1998; Brandi et al 2002; Staniforth et al 2005). However, in comparison with calcitriol, paricalcitol has a decreased effect in intestinal calcium absorption and bone mobilization (Coyne et al 2002). Animal studies in uremic rats found that paricalcitol maintained effective PTH suppression, but had decreased expression of markers for intestinal calcium transport (Brown et al 2002).

Clinical trials have found similar results (Martin et al 1998b; Llach and Yudd 2001; Martin 2001; Sprague 2001). In randomized, double-blind, placebo-controlled clinical trials, paricalcitol decreased PTH by $60 \%$ without significant change in calcium levels (Martin et al 1998a). Direct comparisons of paricalcitol and calcitriol in the hemodialysis population have shown that paricalcitol achieves equivalent PTH suppression at a paricalcitol to calcitriol ratio of approximately 3:1 (Martin et al 1998b; Llach and Yudd 2001). Furthermore, paricalcitol had a lower calciumphosphorus product than calcitriol even at doses 6-8-fold higher than calcitriol (Coyne et al 2002). Paricalcitol is also associated with a lower mortality (Teng et al 2003) in a 
historical cohort study, and lower hospitalization rate (Dobrez et al 2004) in retrospective analyses, a benefit that appeared to be independent of calcium, phosphorus, and PTH levels in comparison with calcitriol.

\section{Oral paricalcitol}

The data with intravenous paricalcitol suggest that this vitamin D analog is beneficial in controlling SHPT in the dialysis population, maintaining a safer effect on calcium and phosphorus levels while associated with improvement in morbidity and mortality when compared with calcitriol. However, the administration of the intravenous formulation is not convenient in patients without the regular intravenous access provided with hemodialysis, such as those on peritoneal dialysis and patients with CKD not yet on dialysis. Paricalcitol capsules were thus developed and recently approved by the US Food and Drug Administration (FDA) in May 2005 for use in the CKD population.

The oral formulation has the same structural modifications and mechanism of action as the intravenous form. It is metabolized by both hepatic and nonhepatic enzymes (including CYP24), and it is eliminated primarily through the hepatobiliary route. It can be dosed daily at a starting dose of $1 \mathrm{mcg}$ in individuals with PTH $<500 \mathrm{pg} / \mathrm{mL}$ and $2 \mathrm{mcg}$ in individuals with PTH $>500 \mathrm{pg} / \mathrm{mL}$. Thrice weekly dosing is also highly effective. It is well absorbed and reaches a peak concentration approximately 3 hours after ingestion. Oral paricalcitol is generally well tolerated. Its main side effects are related to gastrointestinal discomfort. In a recent placebo controlled study, $6 \%$ of patients on paricalcitol experienced nausea (vs $4 \%$ with placebo) and 6\% experienced vomiting (vs $4 \%$ with placebo) (Coyne et al 2006).

Randomized trials have been conducted showing that paricalcitol capsules demonstrate effective suppression of PTH, achieving $\geq 30 \%$ reduction in PTH on 2 consecutive measurements more frequently than those treated with placebo (91\% vs $13 \%, \mathrm{p}<0.001)$ (Coyne et al 2006). Furthermore there was no significant difference between oral paricalcitol and placebo in the incidence of hypercalcemia (>10.5 mg/dL) (2\% vs $0 \%$ respectively, $\mathrm{p}=0.237)$, hyperphosphatemia $(>5.5 \mathrm{mg} / \mathrm{dL})(10 \%$ vs $12 \%$ respectively, $\mathrm{p}=0.830$ ) or elevated calcium-phosphorus products $\left(>55 \mathrm{mg}^{2} / \mathrm{dL}^{2}\right)(12 \%$ vs $6 \%$ respectively, $\mathrm{p}=0.161)$.

Thus far there are no studies comparing oral paricalcitol with other oral active vitamin $\mathrm{D}$ preparations in the predialysis CKD population. In placebo-controlled studies, calcitriol (Slatopolsky et al 1984) and the three currently available vitamin D analogs, alfacalcidol (Hamdy et al 1995), doxercalciferol (Coburn et al 2004), and paricalcitol (Coyne et al 2006), all suppress PTH levels. A study in CKD patients showed that oral calcitriol suppressed baseline PTH levels by $25 \%$ over 1 year of treatment, but at doses between $0.25 \mathrm{mcg} /$ day and $1 \mathrm{mcg} /$ day, calcitriol led to hypercalcemia in $64 \%$ of patients in the trial and a $139 \%$ increase in urinary calcium excretion after 1 year (Calcitriol new drug application [NDA] 18-044 FDA). Additional studies have shown that while low doses of calcitriol are generally well tolerated in the CKD population, the risk of hypercalcemia increases when calcitriol dose exceeds $0.5 \mathrm{mcg}$ /day (Nordal and Dahl 1988; Baker et al 1989). The vitamin D analogs alfacalcidol (1-alpha D3) and doxercalciferol (1-alpha D2) have also demonstrated significant PTH suppression (28\% and $46 \%$ respectively) though hypercalcemia occurred in $15 \%$ of patients treated with alfacalcidol and $4 \%$ of those on doxercalciferol (Hamdy et al 1995; Coburn et al 2004). Urinary calcium concentrations increased by $41 \%$ and $42 \%$ respectively, reflecting increased calcium load. In comparison, paricalcitol capsules had a mean PTH suppression of $42 \%$ with only $2 \%$ of patients in the paricalcitol groups experiencing hypercalcemia (Coyne et al 2006). Mean urinary calcium increased by $6 \%$ and mean serum calcium increased about $1 \%$. Indirect comparison of these results suggests paricalcitol effectively suppressed PTH with smaller alterations in serum calcium and phosphorus.

\section{Potential long term benefits}

It is known that persistent PTH stimulation results in nodular hyperplasia which may not regress even in the face of normalized renal function (ie, after renal transplantation) (McCarron et al 1982). Prolonged exposure to elevated PTH levels may lead to irreversible parathyroid hyperplasia or significant bone disease by the time patients reach end stage renal disease (ESRD). Based on the evidence presented above, oral paricalcitol is able to effectively control PTH secretion in CKD patients when compared with placebo. Early treatment with oral paricalcitol can thus attenuate the development of SHPT and prevent progression to severe forms of disease in the parathyroid gland and bone.

Paricalcitol has also been associated with benefits in patient survival, hospitalizations, and mortality rates in dialysis patients across all levels of serum calcium, phosphorus, or PTH, suggesting that this benefit is not solely attributable to vitamin D's traditional role in bone and mineral homeostasis (Teng et al 2003, 2005; Dobrez et al 
2004). Patients treated with paricalcitol had a 16\% lower mortality rate than those treated with calcitriol in a historical cohort study of over 67000 hemodialysis patients (Teng et al 2003). This survival advantage was also seen in patients who switched from calcitriol to paricalcitol when compared with those who switched from paricalcitol to calcitriol. A separate study found that patients treated with paricalcitol also had decreased hospitalizations and fewer hospital days per year than those treated with calcitriol (Dobrez et al 2004).

The activation of vitamin $\mathrm{D}$ receptors by paricalcitol may mediate a number of benefits independent of its effects on mineral metabolism. Vitamin D receptors are dispersed throughout the body in a number of tissues, and activation of these receptors may affect not only secretion of PTH but also modulation of the renin-angiotensin system, left ventricular hypertrophy, and vascular calcification (Levin and Li 2005). Activation of VDRs may have a role in preserving renal function as well. In animal models, the administration of calcitriol attenuated the development of renal fibrosis in mice models through suppressing transforming growth factor $\beta$ (TGF $\beta$ ) and inducing hepatocyte growth factor (Li et al 2005), an important cytokine that attenuates interstitial fibrosis by inhibiting myofibroblast activation (Yang and Liu 2002). Preliminary studies with paricalcitol show that intravenous paricalcitol decreases renal interstitial fibrosis in mouse models of obstructive nephropathy (Tan and Liu 2005), and oral paricalcitol has demonstrated a protective effect on residual renal function in the CKD population by reducing proteinuria independent of the use of renin-angiotensin blocking agents (Agarwal et al 2005). Further clinical trials are needed to investigate the additional benefits of oral paricalcitol in CKD patients and whether it impacts morbidity, mortality, and time to dialysis.

\section{Pharmacoeconomics}

The cost of care for the management of SHPT is significant. Short term costs include the cost of medications, routine blood work to monitor calcium and phosphorus, and the management of adverse effects. Despite its higher cost, paricalcitol has a lower incidence of hypercalcemia, which may translate into fewer adverse effects and a decreased need for frequent checks of calcium and phosphorus levels. Long term costs include cost of care for hospitalizations and treatment of significant bone disease and cardiovascular events associated with untreated SHPT. Early administration of oral paricalcitol may provide effective control of SHPT without necessitating dose reductions for hypercalcemia or hyperphosphatemia, thus preventing the progression of bone disease and parathyroid hyperplasia in SHPT, leading to fewer hospitalizations. The effect of paricalcitol on preservation of renal function would also reduce the cost of care substantially. Paricalcitol may increase the time to dialysis in CKD patients, thus generating substantial savings. Additionally, the cost of management of SHPT among dialysis patients is likely to be dramatically less if parathyroid hyperplasia is reduced during the CKD period.

\section{Conclusion}

Treatment of SHPT continues to be an important part of care in the patient with CKD. Paricalcitol is a well tolerated and effective medication for reducing PTH levels in the CKD population while minimizing hypercalcemia and hyperphosphatemia. Paricalcitol capsules permit early intervention to halt SHPT and reverse bone-related disease, especially in those patients who are unable to take calcitriol due to hypercalcemia and hyperphosphatemia. However, the benefits of paricalcitol likely extend beyond its effects on renal mineral metabolism. Clinical trials with intravenous paricalcitol show a survival advantage and a decrease in hospitalizations when compared to calcitriol, while animal studies suggest that paricalcitol attenuates vascular calcification, atherosclerosis, and renal fibrosis. This may translate into substantial decreases in the cost of care of patients with kidney disease and SHPT. Further studies will be needed to examine the role of this vitamin $\mathrm{D}$ analog in preventing vascular calcification, preserving renal function, and limiting left ventricular hypertrophy.

\section{References}

Agarwal R, Acharya M, Tian J, et al. 2005. Antiproteinuric effect of oral paricalcitol in chronic kidney disease. Kidney Int, 68:2823-8.

Baker LR, Abrams L, Roe CJ, et al. 1989. 1,25(OH)2D3 administration in moderate renal failure: A prospective double blind trial. Kidney Int, 35:661-9.

Barrat F, Cua D, Boonstra A, et al. 2002. In vitro generation of interleukin 10-producing regulatory CD4+ $\mathrm{T}$ cells is induced by immunosuppressive drugs and inhibited by T helper type (Th1) and Th2 inducing cytokines. J Exp Med, 195:603-16.

Bellows C, Reimers S, Heersche J. 1999. Expression of mRNAs for type 1 collages, bone sialoprotein, osteocalcin and osteopontin at different stages of osteoblastic differentiation and their regulatikons by $1,25-$ dihydroxyvitamin D. Cell Tissue Res, 297:249-59.

Boonstra A, Barrat FJ, Crain C, et al. 2001. 1-alpha-25-dihydroxyvitamin D3 has direct effect on naïve CD4+ T cells to enhance the development of Th2 cells. J Immunol, 167:4974-80.

Brandi L, Egfjord M, Olgaard K. 2002. Pharmacokinetics of 1,25 (OH)2D3 and $1(\mathrm{OH}) \mathrm{D} 3$ in normal and uraemic men. Nephrol Dial Transplant, $17: 829-42$. 
Brown AJ, Finch J, Slatopolsky E. 2002. Differential effects of 19-nor1,25-dihydroxyvitamin D2 and 1,25-dihydroxyvitamin D3 on intestinal calcium and phosphorus transport. J Lab Clin Med, 139:27984

Cato A 3rd, Cady WW, Soltanek C, et al. 1998. Effect of hemodialysis on the pharmacokinetics of 19-nor-1alpha,25-dihydroxyvitamin D2. Am J Kidney Dis, 32:S55-60.

Coburn J, Maung H, Elangovan L, et al. 2004. Doxercalciferol safely suppresses PTH levels in patients with secondary hyperparathyroidism associated with chronic kidney disease stages 3 and 4. Am J Kidney Dis, 43:877-90.

Coyne, D, Charya M, Qiu P, et al. 2006. Paricalcitol capsule for the treatment of secondary hyperparathyroidism in stages 3 and 4 CKD. Am J Kidney Dis, 47:263-76.

Coyne DW, Grieff M, Ahya SN, et al. 2002. Differential effects of acute administration of 19-nor-1,25-dihydroxyvitamin D2 and 1,25dihydroxyvitamin D3 on serum calcium and phosphorus in hemodialysis patients. Am J Kidney Dis, 40:1283-8.

Dobrez D, Mathes A, Amdahl M, et al. 2004. Paricalcitol-treated patients experience improved hospitalization outcomes compared with calcitriol-treated patients in real-world clinical settings. Nephrol Dial Transplant, 19:1174-81.

Drissi H, Pouliot A, Koolloos C, et al. 2002. 1,25-(OH)2-vitamin D3 suppresses the bone-related Runx2/Cbfa2 gene promoter. Exp Cell Res, 274:323-33.

Dusso AS, Brown AJ. 1998. Mechanisms of vitamin D action and its regulation. Am J Kidney Dis, 32(Suppl):13-24.

Goodman WG, Goldin J, Kuizon BD, et al. 2003. Coronary-artery calcifications in young adults with end-stage renal disease who are undergoing dialysis. N Engl J Med, 342:1478-83.

Goldsmith D, Ritz E, Covic A. 2004. Vascular calcification: a stiff challenge for the nephrologist; Does preventing bone disease cause arterial disease? Kidney Int, 66:1315-33.

Hamdy N, Kanis J, Beneton M, et al. 1995. Effect of alfacalcidol on natural course of renal bone disease in mild and moderate renal failure. $B M J$, 310:358-63.

Kramer H, Toto R, Peshock R, et al. 2005. Association between chronic kidney disease and coronary artery calcification: The Dallas Heart Study. J Am Soc Nephrol, 16:507-13.

Levin A, Li Y. 2005. Vitamin D and its analogues: do they protect against cardiovascular disease in patients with kidney disease? Kidney Int, 68:1973-81.

Li Y, Spataro B, Yang J, et al. 2005. 1,25-dihydroxyvitamin D3 inhibits renal interstitial myofibroblast activation by inducing hepatocyte growth factor expression. Kidney Int, 68:1500-10.

Llach F, Yudd M. 2001. Paricalcitol in dialysis patients with calcitriolresistant secondary hyperparathydoisim. Am J Kidney Dis, 38(Suppl):S45-50.

Martin KJ. 2001. Paricalcitol dosing according to body weight or severity of hyperparathyroidism: a double blind, multicenter, randomized study. Am J Kidney Dis, 38(Suppl):S57-63.

Martin KJ, Gonzalez EA, Gellens M, et al. 1998a. 19-Nor-1-alpha-25dihydroxyvitamin D2 (paricalcitol) safely and effectively reduces the levels of intact parathyroid hormone in patients on hemodialysis. $J$ Am Soc Nephrol, 9:1427-32.

Martin KJ, Gonzalez EA, Gellens ME, et al. 1998b. Therapy of secondary hyperparathyroidism with 19-nor-1alpha, 25-dihydroxyvitamin D2. Am J Kidney Dis, 32(2 Suppl 2):S61-6.
McCarron DA, Muthers RS, Lenfesty B, et al. 1982. Parathyroid function in persistent hyperparathyroidism: relationship to gland size. Kidney Int, 22:662-70.

Mee A, Davenport L, Hoyland J, et al. 1996. Novel and sensitive detection systems for the vitamin D receptor - in situ reverse transcriptasepolymerase chain reaction and immunogold cytochemistry. $J \mathrm{Mol}$ Endocrinol, 16:183-96.

Nordal KP, Dahl E. 1988. Low dose calcitriol versus placebo in patients with predialysis chronic renal failure. J Clin Endocrinol Metab, 67:929-36.

Pitts TO, Piraino BH, Mitro R, et al. 1988. Hyperparathyroidism and 1, 25-dihydroxyvitamin D deficiency in mild, moderate, and severe renal failure. J Clin Endocrinol Metab, 67:876-81.

Qunibi WY, Nolan CA, Ayus JC. 2002. Cardiovascular calcification in patients with end-stage renal disease: a century-old phenomenon. Kidney Int Supplemental, 82:73-80.

Reichel H, Deibert B, Schmidt-Gayk H, et al. 1991. Calcium metabolism in early chronic renal failure: Implications for the pathogenesis of hyperparathyroidism. Nephrol Dial Transplant, 6:162-9.

Russell J, Lettieri D, Sherwood LM. 1986. Suppression by 1, 25 (OH) D3 of transcription of the pre-proparathyroid hormone gene. Endocrinology, 119:2864-6.

Sigris M, Bungay P, Taal M, et al. 2006. Vascular calcification and cardiovascular function in chronic kidney disease. Nephrol Dial Transplant, 21:707-14.

Silver J, Naveh-Many T, Mayer H, et al. 1986. Regulation by vitamin D metabolites of parathyroid hormone gene transcription in vivo in the rat. J Clin Invest, 78:1296-301.

Slatopolsky E, Weerts C, Thielan J, et al. 1984. Marked suppression of secondary hyperparathyroidism by intravenous administration of 1,25dihydroxycholecalciferol in uremic patients. J Clin Invest, 74:213643.

Sprague SM. 2001. Suppression of parathyroid hormone secretion in hemodialysis patients: comparison of paricalcitol with calcitriol. Am J Kidney Dis, 38(Suppl):S51-6.

Staniforth M, Cheng S, Coyne D. 2005. Once weekly intravenous paricalcitol in the treatment of secondary hyperparathyroidism in hemodialysis patients. Clin Nephrol, 63:454-60.

Staeva-Vieira T, Freedman L. 2002. 1,25-dihydroxyvitamin D inhibits IFNgamma and IL-4 levels during in vitro polarization of primary murine CD4+ T cells. J Immunol, 168:1181-9.

Tan X, Liu Y. 2005. Paricalcitol attenuates renal interstitial fibrosis in mouse models of obstructive nephropathy [poster]. The Annual Meeting of the American Society of Nephrology, 11-14 November, Philadelphia, PA, USA. FPO422

Teng M, Wolf M, Lowrie E, et al. 2003. Survival of patients undergoing hemodialysis with paricalcitol or calcitriol therapy. New Engl J Med, 349:446-56.

Teng M, Wolf M, Ofsthun M, et al. 2005. Activated injectable vitamin D and hemodialysis survival: A historical cohort study. J Am Soc Nephrol, 16:1115-25.

Virdi AS, Cook LJ, Oreffo RO, et al. 1998. Modulation of bone morphogenetic protein-2 and bone morphogenetic protein-4 gene expression in osteoblastic cell lines. Cell Mo Biol, 44:1237-46.

Yang J, Liu Y. 2002. Blockage of tubular epithelial to myofibroblast transition by hepatocyte growth factor prevents renal interstitial fibrosis. J Am Soc Nephrol, 13:96-107. 
